\title{
Enrichment in conservative amino acid changes among fixed and standing missense variations in slow evolving proteins
}

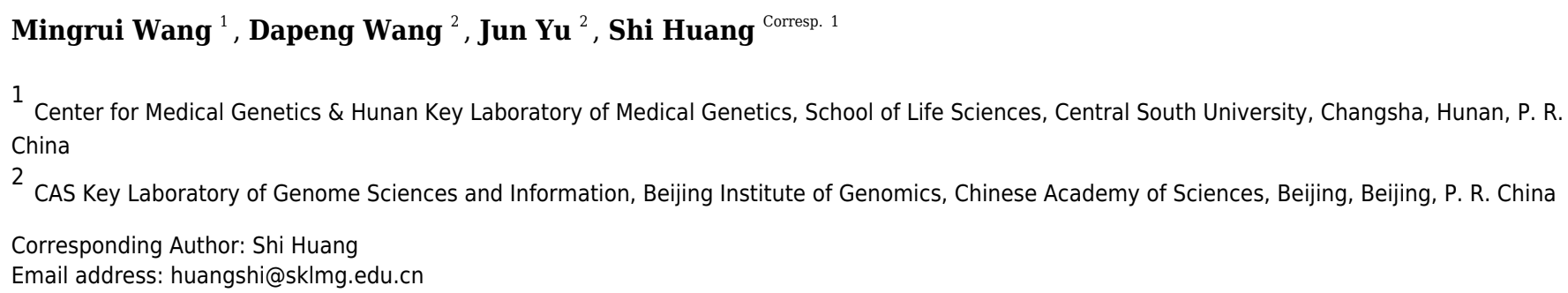

The process of molecular evolution has many elements that are not yet fully understood. Evolutionary rates are known to vary among protein coding and noncoding DNAs, and most of the observed changes in amino acid or nucleotide sequences are assumed to be non-adaptive by the neutral theory of molecular evolution. However, it remains unclear whether fixed and standing missense changes in slowly evolving proteins are more or less neutral compared to those in fast evolving genes. Here, based on the evolutionary rates as inferred from identity scores between orthologs in human and Rhesus Macaques (Macaca mulatta), we found that the fraction of conservative substitutions between species was significantly higher in their slowly evolving proteins. Similar results were obtained by using four different methods of scoring conservative substitutions, including three that remove the impact of substitution probability, where conservative changes require fewer mutations. We also examined the single nucleotide polymorphisms (SNPs) by using the 1000 genomes project data and found that missense SNPs in slowly evolving proteins also had a higher fraction of conservative changes, especially for common SNPs, consistent with more non-conservative substitutions and hence stronger natural selection for SNPS, particularly rare ones, in fast evolving proteins. These results suggest that fixed and standing missense variants in slowly evolving proteins are more likely to be neutral. 
1 Enrichment in conservative amino acid changes among fixed and standing missense

2 variations in slowly evolving proteins

3

4 Mingrui Wang ${ }^{1}$, Dapeng Wang ${ }^{2,3}$, Jun $\mathrm{Yu}^{2}$, and Shi Huang ${ }^{1^{*}}$

5

6 1Center for Medical Genetics \& Hunan Key Laboratory of Medical Genetics, School of Life

7 Sciences, Central South University, Changsha, Hunan 410078, P. R. China

8

$9{ }^{2}$ CAS Key Laboratory of Genome Sciences and Information, Beijing Institute of Genomics,

Chinese Academy of Sciences, Beijing 100029, P.R. China

11

12

*Corresponding author: huangshi@sklmg.edu.cn

13

3Present address: LeedsOmics, University of Leeds, Leeds LS2 9JT, UK

15

Running title: Conservative amino acid changes in slowly evolving proteins 
19

20

21

22

23

\section{Abstract}

The process of molecular evolution has many elements that are not yet fully understood. Evolutionary rates are known to vary among protein coding and noncoding DNAs, and most of the observed changes in amino acid or nucleotide sequences are assumed to be non-adaptive by the neutral theory of molecular evolution. However, it remains unclear whether fixed and standing missense changes in slowly evolving proteins are more or less neutral compared to those in fast evolving genes. Here, based on the evolutionary rates as inferred from identity scores between orthologs in human and Rhesus Macaques (Macaca mulatta), we found that the fraction of conservative substitutions between species was significantly higher in their slowly evolving proteins. Similar results were obtained by using four different methods of scoring conservative substitutions, including three that remove the impact of substitution probability, where conservative changes require fewer mutations. We also examined the single nucleotide polymorphisms (SNPs) by using the 1000 genomes project data and found that missense SNPs in slowly evolving proteins also had a higher fraction of conservative changes, especially for common SNPs, consistent with more non-conservative substitutions and hence stronger natural selection for SNPs, particularly rare ones, in fast evolving proteins. These results suggest that fixed and standing missense variants in slowly evolving proteins are more likely to be neutral. 


\section{Introduction}

Since the early 1960s, protein sequence comparisons have become increasingly important in molecular evolutionary research (Doolittle \& Blombaeck 1964; Fitch \& Margoliash 1967; Margoliash 1963; Zuckerkandl \& Pauling 1962). An apparent relationship between protein sequence divergence and time of separation led to the molecular clock hypothesis, which assumes a constant and similar evolutionary rate among species (Kumar 2005; Margoliash 1963; Zuckerkandl \& Pauling 1962). Thus, sequence divergence between species is thought to be largely a function of time. The molecular clock, in turn, led Kimura to propose the neutral theory to explain nature: sequence differences between species were thought to be largely due to neutral changes rather than adaptive evolution (Kimura 1968). However, the notion of a molecular clock may be unrealistic since it predicts a constant substitution rate as measured in generations, whereas the observed molecular clock is measured in years (Ayala 1999;

Pulquerio \& Nichols 2007). The neutral theory remains an incomplete explanatory theory (Hu et al. 2013; Kern \& Hahn 2018).

1 Evolutionary rates are known to vary among protein coding and non-coding DNAs. The neutral theory posits that the substitution rate under selective neutrality is expected to be equal to the mutation rate (Kimura 1983). If mutations/substitutions are not neutral or are under natural selection, the substitution rate would be affected by the population size and the selection coefficient, which are unlikely to be constant among all lineages. Slowly evolving genes are well known to be under stronger purifying or negative selection as measured by using $\mathrm{dN} / \mathrm{dS}$ ratio, which means that a new mutation has a lower probability of being fixed (Cai \& Petrov 2010). 
However, negative selection as detected by the dN/dS method is largely concerned with nonobserved mutations and says little about the fixed or observed variations. And most molecular evolutionary approaches such as phylogenetic and demographic inferences are concerned with observed variants. It remains to be determined whether fixed and standing missense substitutions in slowly evolving genes are more or less neutral relative to those in fast evolving genes.

We here examined the fraction of conservative substitutions (amino acid replacement in a protein that changes a given amino acid to a different amino acid with similar biochemical properties) in proteins of different evolutionary rates. We compared the protein orthologs of two relatively closely related species, Homo sapiens and Macaca mulatta, to obtain values of percentage identity to represent evolutionary rates. We found that the proportion of conservative substitutions between species was higher in the slowest evolving set of proteins than in faster evolving proteins. Using datasets from the 1000 genomes (1KG) project phase 3 dataset (Auton et al. 2015), we also found that missense single nucleotide polymorphisms (SNPs) from the slowest evolving set of proteins, especially those with high minor allele frequency (MAF), were enriched with conservative amino acid changes, consistent with these changes being under weaker natural selection.

\section{Materials and Methods}

Classification of proteins as slowly and fast evolving. The identification of slowly evolving proteins and their associated SNPs was done as previously described (Yuan et al. 
79

80

81

82

2017). Briefly, we collected the whole genome protein data of Homo sapiens (version 36.3) and Macaca mulatta (version 1) from the NCBI FTP site, and then compared the human protein to the monkey protein using local BLASTP program at a cutoff of 1E-10. We only retained one human protein with multiple isoforms, and chose the monkey protein with the most significant Evalue as the orthologous counterpart of each human protein. The aligned proteins were ranked by percentage identities. Proteins that show the highest identity between human and monkey were included in the set of slowly evolving (including 423 genes $>304$ amino acid in length with $100 \%$ identity and 178 genes $>1102$ amino acid in length with $99 \%$ identity between monkey and human). The rest are all considered fast evolving proteins. The cutoff criterion was based on the empirical observation of low substitution saturation, and the finding that missense SNPs from the slow set of proteins produced genetic diversity patterns that were distinct from those found in the fast set (Yuan et al. 2017). The BLASTP alignment program is not expected to produce very different results from other programs, especially for highly conserved proteins. We have limited our analysis to high identity orthologs with length $>200$ amino acid and percent identity $>60 \%$ between monkey and human. So, variation in alignment is not expected to affect comparing our analysis to others.

SNP selection. We downloaded the 1KG phase 3 data and assigned SNP categories using ANNOVAR (Auton et al. 2015). We then picked out the missense SNPs located in the slow evolving set of genes from the downloaded VCF files (Yuan et al. 2017). MAF was derived from AF (alternative allele frequency) values from the VCF files. Missense SNPs in fast evolving genes included all those from 1KG that are not from the slowly evolving set. 
Scoring conservative amino acid replacements. We downloaded the Consensus CDS

101

102

data of Homo sapiens (version 36.3,

https://ftp.ncbi.nlm.nih.gov/pub/CCDS/archive/Hs36.3/CCDS_protein.20090327.faa.gz) and

CDS data of Macaca mulatta (version 1.0, http://ftp.ensembl.org/pub/release-

85/fasta/macaca_mulatta/pep/Macaca_mulatta.MMUL_1.pep.all.fa.gz) and searched for related protein pairs using BLASTP program in BLAST+ 2.10.0. Human proteins were used as queries

to search in the monkey protein database. We recorded the IDs of query and the first hit (ranked by E-values), query length, alignment span, the number of matching amino acids, the combined number of gaps in the query and hit proteins, and the number and type of amino acid substitutions.

For fixed substitutions as revealed by BLASTP, conservative changes were scored by using four different matrixes. The BLOSUM62 matrix has a scoring range from -3 to $3(-3,-2,-1,0,1$, $2,3)$ with higher positive values representing more conservative changes (Pearson 2013). We assigned each amino acid mutation a score and we used score $>0$ to denote conservative changes in cases where the number of conservative changes is enumerated. As the BLOSUM62 matrix does not take into account the effect of substitution probability (the fact that conservative changes require fewer mutations), we also used three other matrixes to score conservative amino acid replacements that have removed the impact of substitution probability, including the "EX" matrix (Yampolsky \& Stoltzfus 2005), which is based on laboratory mutagenesis, and the two physicochemical matrices in Braun (2018) and Pandey and Braun (2020): delta_V (normalized change in amino acid side chain volume), and delta_P (normalized 
121

change in amino acid side chain polarity) (Braun 2018; Pandey \& Braun 2020). All three matrixes in spreadsheets are available from GITHUB

(https://github.com/ebraun68/clade_specific_prot_models). Specifically, the EX matrix (or, more accurately, a normalized symmetric version of the EX matrix) is in the excel spreadsheet "EX_matrix_sym.xlsx"; the delta_V and delta_P matrices can be found in one of the sheets (the sheet called "Exchanges") in the file "exchange_Pandey_Braun.xlsx". All three of the matrixes are normalized to range from zero to one. To be comparable to the BLOSUM62 matrix, we generated integer versions of these three matrixes by multiplying by 10 , subtracting 5 , and then rounding to the nearest integer. Here the matrix values range from -5 to +5 with higher positive values representing more conservative changes. For EX matrix, we used score $>2$ to denote conservative changes. For delta_V and delta_P matrixes, we used score $>3$ to denote conservative changes. In this way of using different cutoff scores to represent conservative changes, we could keep the fraction of conservative changes close to 0.5 for each of the four different matrixes.

Statistics. Chi-squared test was performed using GraphPad Prism 6.

\section{Results:}

\section{Fixed amino acid substitutions and evolutionary rates of proteins}

We determined the evolutionary rates of proteins in the human genome by the percentage of identities between human proteins and their orthologs in Macaca mulatta as described previously (Yuan et al. 2017). We then divided the proteins into several groups of different 
142

143

144

145

146

147

evolutionary rates, and compared the proportion of conservative amino acid substitutions in each group.

The mismatches between two species would have one of the two residues or alleles as ancestral, in the case of slowly evolving proteins yet to reach mutation saturation (no independent mutations occurring at the same site among species and across time), and so a mismatch due to conservative changes would involve a conservative mutation during evolution from the ancestor to extant species. But at mutation saturation for fast evolving proteins, where a site had encountered multiple mutations across taxa and time, while a drastic substitution would necessarily involve a non-conservative mutation, it is possible for a conservative substitution to result from at least two independent non-conservative mutations (if the common ancestor has Arg at some site, a drastic mutation event at this site occurring in each of the two species, Arg to Leu in one and Arg to lle in the other, may lead to a conservative substitution of Leu and lle). Thus, a conservative substitution at mutation saturation just means less physical and chemical differences between the two species concerned and says little about the actual mutation events. A lower fraction of conservative substitutions at saturation for fast evolving proteins would mean more physical and chemical differences between the two species, which may more easily translate into functional differences for natural selection to act upon.

To verify that the slowest evolving proteins with length $>1102$ amino acids and percentage identity $>99 \%$ are distinct from the fast set, we first compared proteins with length $>1102$ amino acids with no gaps in alignment (Table 1, Figure 1A) or with gaps (Table 1, Figure 1B) divided into 4 groups of different percentage identity between human and monkey, >99\%, 98-99\%, 96- 
163

164

165

166

167

168

$98 \%$, and $87-97 \%$. We used four different scoring matrixes to give each amino acid change a rank score in terms of how conservative the change is, BLOSUM62 (Pearson 2013), EX (Yampolsky \& Stoltzfus 2005), delta_V, and delta_P (Braun 2018; Pandey \& Braun 2020). The results were largely similar. There was a general correlation between slower evolutionary rates and higher fractions of conservative changes, with a significant drop in the fraction of conservative changes between the slowest evolving, which was included in the slow set that has monkey-human identity $>99 \%$ and protein length $>1102$ amino acids, and the next slowest set (Figure $1 \mathrm{~A}$ and $\mathrm{B})$. Proteins with alignment gaps showed similar or slightly lower fractions of conservative changes than those without gaps. We further studied the remaining proteins with shorter protein length (200-1102 amino acids) divided into 4 groups (95-99\%, 90-95\%, 80-90\%, and $60-80 \%$ identity), and found similar but less robust and consistent trends (Table 1 and Figure $1 \mathrm{C}$ and $\mathrm{D})$.

\section{Standing amino acid variants and evolutionary rates of proteins}

We next studied the missense SNPs found in proteins with different evolutionary rates by using 1 KG dataset (Auton et al. 2015). There were 15271 missense SNPs in the slowly evolving set of proteins (>1102 aa with $99 \%$ identity and >304 aa with 100\% identity) and 546297 missense SNPs in the fast set (all proteins that remain after excluding the slow set). We assigned each amino acid change found in a missense SNP a conservation score as described above. The number of SNPs in each score category was then enumerated. We performed this analysis by using each of the four different scoring matrixes and found largely similar results 
184 (Figure 2). Missense SNPs in the slowly evolving set of proteins in general had lower fractions

185

186

187

188

189

190

191

192

193

194

195

196

197

198

199

200

201

202

203

204

of drastic mutations, and higher fractions of conservative mutations relative to those in the faster

evolving set of proteins (Figure 2). The fraction of conservative mutations in the slow evolving

set was significantly higher than that of the fast set $(P<0.001$, Figure 2$)$.

To test for natural selection regarding conservative changes, we next divided the slowly

evolving set of missense SNPs into three groups of different minor allele frequency (MAF) as

measured in Africans (similar results were found for other racial groups). For fast evolving

proteins at mutation saturation, low MAF values of a missense SNP would mean stronger

negative selection, and so SNPs with low MAF are expected to have lower proportions of

conservative amino acid changes, since these changes may mean too little functional alteration

to be under natural selection. The results showed that for missense SNPs in the fast evolving

set of proteins, the common SNPs with MAF $>0.001$ showed a higher fraction of conservative

changes than the rare SNPs with MAF $<0.001(P<0.001)$, indicating a stronger natural selection

for the rare SNPs in the fast set (Figure 3). While SNPs in the fast set showed similar fractions

of conservative changes across three different MAF groups $(>0.001,>0.01$, and $>0.05)$, there

was a more obvious trend of having a higher proportion of conservative changes as MAF values

increase from $>0.001$ to $>0.01$ to $>0.05$ for SNPs in the slow set, consistent with weaker natural

selection for common SNPs in the slow set (Figure 3). Each of the three groups in the fast set

showed a significantly lower fraction of conservative changes than the respective group in the

slow set $(P<0.01)$, indicating stronger natural selection for SNPs in the fast set (Figure 3). The

results indicate that common SNPs in slowly evolving proteins had more conservative changes

Peer] reviewing PDF | (2019:12:43889:3:0:NEW 12 Aug 2020) 
205

206

207

208

209

210

211

212

that were under a weaker natural selection.

\section{Discussion:}

Our results here showed that fixed and standing changes in slowly evolving proteins were enriched with conservative amino acid substitutions. Similar results were obtained using four different matrixes to rank the conservative nature of a substitution. Based on substitution probability alone, amino acid substitutions in slowly evolving proteins are expected to be more conserved than those in fast evolving proteins, since fast evolving proteins have a higher probability of the doublet mutations that are necessary for a drastic substitution to occur, but have a very low rate of occurrence (Whelan \& Goldman 2004). If evolutionary time is not long enough for mutation saturation to occur, non-conservative substitutions would be expected to be a function of mutation rate and time. This simple explanation appears not to be the reason for the observations here, since the three matrixes that have removed the impact of substitution probability produced similar results as the matrix that does not take into account the impact of substitution probability.

The four matrixes we used were developed in different ways: the BLOSUM62 matrix by using sequence alignments involving relatively divergent species, the delta_V and delta_P matrixes directly from the physicochemical properties of amino acids, and the EX matrix from experimental mutagenesis. However, the matrixes are still expected to provide information that applies to alignment data from relatively closely related species such as monkey and human, since proteins identified as fast evolving by comparing closely related species would also in 
general be identified as such by comparing more distantly related species. This is suggested by

227 the molecular clock phenomenon or the constant evolutionary rates across time and species. It appears that the delta_V matrix produced less significant results compared to the other three matrixes. Overestimation of the number of conservative substitutions in fast evolving proteins may account for this. For example, substitutions involving differently charged residues with similar side chain volumes would be scored as non-conservative by the delta_P matrix but conservative by the delta_V matrix (e.g., Glu to Leu).

Our analysis does not take into account the co-evolution and co-variation of substitutions due to the physico-chemical constraints on protein structure and folding (Pollock et al. 2012).

Site specific variations in substitution constraints however may be similarly present in different proteins of different evolutionary rates so that they may not affect the overall results here. Also,

Pollock et al. show that site-specific preferences shift over time due to substitutions at other sites that are epistatic to the site of interest (Pollock et al. 2012). Thus, it could be very complex to define site-specific preferences in a meaningful way.

It has recently been shown that coding region mutation rates as measured prior to the effect

of natural selection are significantly lower in genes where mutations are more likely to be deleterious (Monroe et al. 2020). Mutations are more likely to be deleterious and less likely to be fixed in highly conserved proteins, which are by definition more common in slowly evolving proteins. Thus, slowly evolving genes in fact do have inherently slower mutation rates, which would make them less likely to reach mutation saturation. 
where multiple recurrent mutations at the same site have occurred across taxa and time (Figure

4). At saturation, the range of mutations that have happened at any given site of any given

taxon is irrelevant to the particular type of possible alleles the site may carry at present time.

Natural selection is expected to play an important role in determining that. And natural selection of

course would be most efficient if the mutated allele is functionally very different from the non-

mutated allele. If two taxa are different in traits, it would follow that some of the differences in

protein sequence between them would be non-neutral or non-conservative changes. Fast

evolving genes play more adaptive roles and hence are more involved in accounting for the

different traits, and so are expected to be enriched with non-conservative substitutions

compared to slowly evolving genes. A fast evolving and adaptive site is more likely to be

mutated more than once or encounter mutation saturation.

Fixed and standing conservative variants in slowly evolving proteins may be under weaker

natural selection for several reasons. First, substitutions in slowly evolving proteins are more

likely to be conservative and conservative changes may not alter protein structure and function

as dramatically as the drastic changes, which may make it harder for natural selection to occur.

Second, as fixed variants cannot be fixed because of negative selection on the variants per

se, they are either neutral or under positive selection. Indeed, fast evolving proteins are known

to be under more positive selection (Cai \& Petrov 2010; Yuan et al. 2017), which implies that

fixed variants in slowly evolving proteins can only become more neutral. Even if slightly 
268

269

270

271

272

273

274

because a mutation that takes a long time to arrive would be useless for quick adaptive needs.

Finally, SNPs in the slow set may be under negative selection if they produce drastic changes, or under no selection if they produce conservative changes (assuming no positive selection as explained above). While one would expect less conservative changes in the rare SNPs compared to the common SNPs, since negative selection may account in part for the low MAF value, the difference in the fraction of conservative changes between the rare SNPs and the common ones in the slow set should be greater than that in the fast set, since the SNPs in the fast set may be under natural selection regardless of MAF values (low MAF SNPs under more negative selection while high MAF SNPs under both positive and negative selection). Our results are consistent with such expectations.

If substitutions in fast evolving proteins are at saturation and under natural selection as indicated here, it would follow that genetic distances or degrees of sequence mismatches between taxa in these proteins would be at saturation, or no longer correlated exactly with time. It is easy to tell the difference between optimum/maximum saturation genetic distances and linear distances as described previously (Huang 2010). Briefly, imagine a 100 amino acid protein with only 1 neutral site. In a multispecies alignment involving at least three taxa, if one finds only one of these taxa with a mutation at this neutral site while all other species have the same non-mutated residue, there is no saturation (Figure 4, time point 2). However, if one finds that nearly every taxon has a unique amino acid, one would conclude mutation saturation as there would have been multiple independent substitution events among different species at the same site, and repeated mutations at the same site do not increase distance (Figure 4, time 
289

290

291

292

293

294

295

296

297

298

299

300

301

302

303

304

305

306

307

308

309

point 3 and 4 for fast evolving proteins). We have termed those sites with repeated mutations

"overlap" sites (Huang 2010). So, a diagnostic criterion for saturated maximum distance

between two species is the proportion of overlap sites among mismatched sites. Saturation

would typically have $50-60 \%$ overlapped sites that are $2-3$ fold higher than that expected before

saturation (Huang 2010; Luo \& Huang 2016). It is not expected to have near $100 \%$ overlapped

sites, because certain sites may only accommodate 2 or very few amino acid residues at

saturation equilibrium, which would prevent them from presenting as overlapped sites even

though they are in fact overlapped and saturated sites. Also, saturation may result in convergent

evolution with independent mutations changing to the same amino acid (Figure 4, time point 5

for fast evolving proteins). This overlap ratio method is an empirical one free of uncertain

assumptions and hence more realistic than other methods of testing for saturation, such as

comparing the observed number of mutations to the inferred one based on uncertain

phylogenetic trees derived from maximum parsimony or maximum likelihood methods (Philippe

et al. 1994; Steel et al. 1993; Xia et al. 2003).

By using the overlap ratio method, we have verified that the vast majority of proteins show

maximum distances between any two deeply diverged taxa, and only a small proportion, the

slowest evolving, are still at the linear phase of changes (Huang 2010; Luo \& Huang 2016; Yuan

et al. 2017). Variations at most genomic sites within human populations are also at optimum

equilibrium, as evidenced by the observation that a slight increase above the present genetic

diversity level in normal subjects is associated with patient populations suffering from complex

diseases (Gui et al. 2017; He et al. 2017; Lei \& Huang 2017; Lei et al. 2018; Yuan et al. 2012;

Peer) reviewing PDF | (2019:12:43889:3:0:NEW 12 Aug 2020) 
310 Yuan et al. 2014; Zhu et al. 2015), as well as the observation that the sharing of SNPs among

311 different human groups is an evolutionary rate-dependent phenomenon, with more sharing in

312 fast evolving sequences (Yuan et al. 2017). It is important to note that a protein in a complex

313 species plays more roles than its orthologous protein in a species of less organismal complexity,

314 as explained by the maximum genetic diversity hypothesis (Hu et al. 2013; Huang 2008; Huang

2016). A protein has more functions to play in complex organisms due in part to its involvement

in more cell types, and hence it becomes more susceptible to mutational inactivation. While the

divergence time among higher taxa such as between human and Macaca monkey is relatively

short, mutation saturation could still happen for fast evolving proteins since the number of

positions that can accept fixed substitutions is comparatively lower.

It is also important to note that the type of saturation we describe here is slightly different

from that seen in "long branch attraction (LBA)" in phylogenetic trees (Bergsten 2005). In LBA,

saturation means convergent mutations leading to the same amino acid residue or nucleotide

among (across) multiple taxa (Figure 4, time point 5 for fast evolving protein P1 and P2).

Although they were derived independently, these shared alleles can be misinterpreted in

phylogenetic analyses as being shared due to common ancestry. However, for the type of

saturation we have discussed here, independent mutations at the same site among different

taxa would generally lead to different taxa having different amino acids rather than the same

(Figure 4, time point 3 and 4 for fast evolving proteins), since the probability of an independent

mutation changing to the same amino acid is about 20 times lower than that of mutating to a 
331

332

amino acids). Thus, the type of saturation we have described here is expected to be more commonplace in nature compared to that in the case of LBA. Since a single mutation is sufficient for a mismatch between any two taxa, multiple independent mutations at the same site leading to different amino acids would not increase the number of mismatches and would remain unnoticeable if one only aligns the sequences from two different taxa (Figure 4, the number of mismatch between P2 and P3 is 1 at time point 2 before saturation and remains as 1 at time point 4 after saturation). It only becomes apparent when one aligns the sequences from three different taxa (Figure 4, time point 3 and 4 for fast evolving proteins), as we described above and in previous publications (Huang 2010; Luo \& Huang 2016). However, even though the type of saturation we describe here does not increase the number of mismatches, it could result in a reduced number of mismatches in rare cases when independent mutations in two different taxa happen to lead to the same residue (Figure 4, time point 5 for P1 and P2). Thus, it does not preclude the type of saturation observed in the case of LBA. These two types of saturation are essentially just two different aspects of the same saturation phenomenon, one more commonplace and manifesting as a higher overlap ratio while the other less common and manifesting as LBA.

It is well known that fast evolving proteins that have reached mutation saturation are not suitable for phylogenetic inferences. We have previously shown that mutation saturation as measured by the overlap ratio method has been largely overlooked (Huang 2012; Yuan et al. 2017), in contrast to the long noted LBA. As mentioned above, it appears that the inherent mutation rates are different between fast and slowly evolving proteins as determined by 
352

353

354

355

356

357

358

359

360

361

362

363

364

365

366

367

368

369

370

371

372

studying the rate of fixed substitutions (Monroe et al. 2020). We can thus infer that if the rate difference is large enough, slowly evolving genes should be used in phylogenetic inferences because they would be less likely to reach mutation saturation. The findings here that fast evolving proteins are enriched with non-neutral substitutions relative to slowly evolving proteins are consistent with such an idea.

There are two points to note regarding fast and slowly evolving proteins. First, the definition of slowly evolving proteins here (99\% identity) is only meant for the specific comparison between human and monkey. For relatively more distantly related species such as human and mouse, the set of slowly evolving proteins is expected to be similar but the percentage identity cutoff for the slow set would be lower than $99 \%$. This is because proteins are known to evolve at constant rates across time and species according to the molecular clock and the neutral theory.

Second, the classification of fast evolving proteins is not absolute and is evolutionary timedependent. Proteins that are found as fast evolving or have reached mutation saturation after a certain relatively long time of evolution are expected to look like slowly evolving or not showing mutation saturation if evolutionary time is relatively short. This is supported by our results here of a nearly linear relationship between evolutionary rates and the fraction of non-conservative changes.

Our finding supports the possibility that, from early on since first diverging from a common ancestor, two sister species are expected to accumulate mostly neutral mismatches, which would later be replaced by non-conservative mismatches when time is long enough for mutation saturation to have taken place. This is to be expected as sister species should become more 
373

374

375

376

377

378

379

380

381

382

383

384

385

386

387

388

389

390

391

392

393

394

395

396

397

differentiated in phenotypes with time, and hence more different in sequences with time in terms

of both the number of mismatches as well as the chemical nature (conservative or not) of the mismatches.

\section{Conclusion:}

Our study here addressed whether observed amino acids variants in slowly evolving

proteins are more or less neutral than those in fast evolving proteins. The results suggest that

fixed and standing missense variations in slowly evolving proteins are more likely to be neutral, and have implications for phylogenetic inferences.

\section{Declarations:}

\section{Acknowledgements:}

We thank the editor and reviewers for their valuable constructive advice and comments.

\section{References:}

Auton A, Brooks LD, Durbin RM, Garrison EP, Kang HM, Korbel JO, Marchini JL, McCarthy S, McVean GA, and Abecasis GR. 2015. A global reference for human genetic variation. Nature 526:68-74. 10.1038/nature15393

Ayala FJ. 1999. Molecular clock mirages. Bioessays 21:71-75.

Bergsten J. 2005. A review of long-branch attraction. Cladistics 21:163-193.

Braun EL. 2018. An evolutionary model motivated by physicochemical properties of amino acids reveals variation among proteins. Bioinformatics 34:i350-i356. 10.1093/bioinformatics/bty261

Cai JJ, and Petrov DA. 2010. Relaxed purifying selection and possibly high rate of adaptation in primate lineage-specific genes. Genome Biol Evol 2:393-409. 10.1093/gbe/evq019 
398

399

400

401

402

403

404

405

406

407

408

409

410

411

412

413

414

415

416

417

418

419

420

421

422

423

424

425

426

427

428

429

430

431

432

433

434

435

436

437

438

Doolittle RF, and Blombaeck B. 1964. Amino-Acid Sequence Investigations Of Fibrinopeptides From Various Mammals: Evolutionary Implications. Nature 202:147-152.

Fitch WM, and Margoliash E. 1967. Construction of phylogenetic trees. Science 155:279-284.

Gui Y, Lei X, and Huang S. 2017. Collective effects of common SNPs and genetic risk prediction in type 1 diabetes. Clin Genet 93:1069-1074. 10.1111/cge.13193

He P, Lei X, Yuan D, Zhu Z, and Huang S. 2017. Accumulation of minor alleles and risk prediction in schizophrenia. Sci Rep 7:11661. 10.1038/s41598-017-12104-0

Hu T, Long M, Yuan D, Zhu Z, Huang Y, and Huang S. 2013. The genetic equidistance result, misreading by the molecular clock and neutral theory and reinterpretation nearly half of a century later. Sci China Life Sci 56:254-261.

Huang S. 2008. Inverse relationship between genetic diversity and epigenetic complexity. Nature Precedings:doi.org/10.1038/npre.2009.1751.1032.

Huang S. 2010. The overlap feature of the genetic equidistance result, a fundamental biological phenomenon overlooked for nearly half of a century. Biological Theory 5:40-52.

Huang S. 2012. Primate phylogeny: molecular evidence for a pongid clade excluding humans and a prosimian clade containing tarsiers. Sci China Life Sci 55:709-725.

Huang S. 2016. New thoughts on an old riddle: What determines genetic diversity within and between species? Genomics 108:3-10. 10.1016/j.ygeno.2016.01.008

Kern AD, and Hahn MW. 2018. The Neutral Theory in Light of Natural Selection. Mol Biol Evol 35:13661371. $10.1093 / \mathrm{molbev} / \mathrm{msy} 092$

Kimura M. 1968. Evolutionary rate at the molecular level. Nature 217:624-626.

Kimura M. 1983. The neutral theory of molecular evolution. Cambridge: Cambridge University Press.

Kumar S. 2005. Molecular clocks: four decades of evolution. Nat Rev Genet 6:654-662.

Lei X, and Huang S. 2017. Enrichment of minor allele of SNPs and genetic prediction of type 2 diabetes risk in British population. PLOS ONE 12:e0187644. 10.1371/journal.pone.0187644

Lei X, Yuan J, Zhu Z, and Huang S. 2018. Collective effects of common SNPs and risk prediction in lung cancer. Heredity:doi:10.1038/s41437-41018-40063-41434.

Luo D, and Huang S. 2016. The genetic equidistance phenomenon at the proteomic level. Genomics 108:25-30. 10.1016/j.ygeno.2016.03.002

Margoliash E. 1963. Primary structure and evolution of cytochrome c. Proc Natl Acad Sci 50:672-679.

Monroe JG, Srikant T, Carbonell-Bejerano P, Exposito-Alonso M, Weng M-L, Rutter MT, Fenster CB, and Weigel D. 2020. Mutation bias shapes gene evolution in Arabidopsis thaliana. bioRxiv:doi/10.1101/2020.1106.1117.156752.

Pandey A, and Braun EL. 2020. Protein evolution is structure dependent and non-homogeneous across the tree of life. bioRxiv:doi.org/10.1101/2020.1101.1128.923458.

Pearson WR. 2013. Selecting the Right Similarity-Scoring Matrix. Curr Protoc Bioinformatics 43:3 5 1-9. 10.1002/0471250953.bi0305s43

Philippe H, Sorhannus U, Baroin A, and Perass R. 1994. Comparison of molecular and paleontological data in diatoms suggests a major gap in the fossil record. J Evol Biol 7:247-265.

Pollock DD, Thiltgen G, and Goldstein RA. 2012. Amino acid coevolution induces an evolutionary Stokes shift. Proc Natl Acad Sci U S A 109:E1352-1359. 10.1073/pnas.1120084109

Peer] reviewing PDF | (2019:12:43889:3:0:NEW 12 Aug 2020) 
439

440

441

442

443

444

445

446

447

448

449

450

451

452

453

454

455

456

457

458

459

460

461

462

463

464

465

466

467
Pulquerio MJ, and Nichols RA. 2007. Dates from the molecular clock: how wrong can we be? Trends Ecol Evol 22:180-184.

Steel MA, Lockhart PJ, and Penny D. 1993. Confidence in evolutionary trees from biological sequence data. Nature 364:440-442. 10.1038/364440a0

Whelan S, and Goldman N. 2004. Estimating the frequency of events that cause multiple-nucleotide changes. Genetics 167:2027-2043. 10.1534/genetics.103.023226

Xia X, Xie Z, Salemi M, Chen L, and Wang Y. 2003. An index of substitution saturation and its application. Mol Phylogenet Evol 26:1-7.

Yampolsky LY, and Stoltzfus A. 2005. The exchangeability of amino acids in proteins. Genetics 170:1459-1472. 10.1534/genetics.104.039107

Yuan D, Lei X, Gui Y, Wang M, Zhang Y, Zhu Z, Wang D, Yu J, and Huang S. 2017. Modern human origins: multiregional evolution of autosomes and East Asia origin of $Y$ and mtDNA. bioRxiv:doi.org/10.1101/101410

Yuan D, Zhu Z, Tan X, Liang J, Zeng C, Zhang J, Chen J, Ma L, Dogan A, Brockmann G, Goldmann G, Medina E, Rice AD, Moyer RW, Man X, Yi K, Li Y, Lu Q, Huang Y, Wang D, Yu J, Guo H, Xia K, and Huang S. 2012. Minor alleles of common SNPs quantitatively affect traits/diseases and are under both positive and negative selection. arXiv:12092911.

Yuan D, Zhu Z, Tan X, Liang J, Zeng C, Zhang J, Chen J, Ma L, Dogan A, Brockmann G, Goldmann O, Medina E, Rice AD, Moyer RW, Man X, Yi K, Li Y, Lu Q, Huang Y, and Huang S. 2014. Scoring the collective effects of SNPs: association of minor alleles with complex traits in model organisms. Sci China Life Sci 57:876-888. 10.1007/s11427-014-4704-4

Zhu Z, Man X, Xia M, Huang Y, Yuan D, and Huang S. 2015. Collective effects of SNPs on transgenerational inheritance in Caenorhabditis elegans and budding yeast. Genomics 106:2329. 10.1016/j.ygeno.2015.04.002

Zuckerkandl E, and Pauling L. 1962. Molecular disease, evolution, and genetic heterogeneity, Horizons in Biochemistry. New York: Academic Press. 
468

469

470

471

472

473

474

475

476

477

480

481

482

483

484

485

486

487

488

489

Tables:

Table 1. Relationship between evolutionary rates and the conservative nature of fixed

amino acid substitutions. Evolutionary rates of proteins in the human genome are

represented by the percentage of identities between human proteins and their orthologs in

Macaca monkey. The proteins are divided into groups of different evolutionary rates, and the

proportion of conservative amino acid mismatches in each group are shown for the four different

ranking matrixes. Not all proteins encoded by the macaque and human genomes are

considered because some proteins do not have easily identifiable orthologs. Also, we limited our

analysis to proteins that have length $>200$ amino acids and show $>60 \%$ identity between

macaque and human in order to reduce the chance of misidentifying orthologs.

\section{Figure Legends:}

Figure 1. Fraction of conservative substitutions in fixed changes in proteins of different

evolutionary rates. Shown are fractions of conservative changes in proteins of length $>1102$

aa $(A, B)$ or 200-1102 aa (C, D) with either no gaps in alignment $(A, C)$ and with gaps $(B, D)$. *:

$\mathrm{P}<0.05$, Chi squared test.

Figure 2. Fraction of conservative substitutions in standing missense substitutions in proteins of different evolutionary rates. Missense SNPs from either the slow or the fast group 
490

491

492

493

494

495

496

497

498

499

500

501

502

503

504

505

506

507

508

509

of proteins were classified based on the scores in the four different matrixes as shown in $A, B$,

$C$, and D. Shown are the fractions of each class. ${ }^{* *}, P<0.001 .{ }^{* *}, P<0.01$, Chi squared test.

\section{Figure 3. Fraction of conservative substitutions in missense SNPs with different MAFs in}

proteins of different evolutionary rates. SNPs from either fast or slowly evolving proteins

were classified based on MAF values and the fractions of conservative changes in each class

are shown. Statistical significance score in difference between slow and fast or between

different MAF cutoffs are shown. ${ }^{* *}, P<0.01$. Chi squared test.

\section{Figure 4. Illustration of non-conservative substitutions and mutation saturation in fast}

evolving proteins. Three orthologous proteins from three different taxa are shown for either

fast evolving (designated P1, P2, and P3) or slow evolving proteins (P4, P5, and P6).

Nucleotide codon for residue $E$ and its mutated codon in $P 1, P 3$, and P6 are also shown. A

doublet mutation is found in the non-conservative substitution in P3 at time point 2, whereas

only a single mutation is found in the conservative substitution in P6. Saturation phase for fast

evolving proteins includes time point 3 to 5 . The type of saturation we describe here is shown at time point 3 and 4 while the type seen in "long branch attraction" is shown at time point 5 . At the saturation phase, for the fast evolving protein $\mathrm{P} 1$ to have the new allele $\mathrm{D}$ at time point 4 or $\mathrm{S}$ at time point 5 is largely a matter of natural selection. 


\section{Table $\mathbf{1}$ (on next page)}

Table 1. Relationship between evolutionary rates and the conservative nature of fixed amino acid substitutions

Evolutionary rates of proteins in the human genome are represented by the percentage of identities between human proteins and their orthologs in Macaca monkey. The proteins are divided into groups of different evolutionary rates, and the proportion of conservative amino acid mismatches in each group are shown for the four different ranking matrixes. Not all proteins encoded by the macaque and human genomes are considered because some proteins do not have easily identifiable orthologs. Also, we limited our analysis to proteins that have length $>200$ amino acids and show $>60 \%$ identity between macaque and human in order to reduce the chance of misidentifying orthologs. 
1 Table 1. Relationship between evolutionary rates and the conservative nature of fixed

2 amino acid substitutions. Evolutionary rates of proteins in the human genome are

3 represented by the percentage of identities between human proteins and their orthologs in

4 Macaca monkey. The proteins are divided into groups of different evolutionary rates, and the

5 proportion of conservative amino acid mismatches in each group are shown for the four different

6 ranking matrixes. Not all proteins encoded by the macaque and human genomes are

7 considered because some proteins do not have easily identifiable orthologs. Also, we limited our

8 analysis to proteins that have length $>200$ amino acids and show $>60 \%$ identity between

9 macaque and human in order to reduce the chance of misidentifying orthologs.

Protein length $>1102$ amino acid with no gaps in alignment

\begin{tabular}{|c|c|c|c|c|c|c|}
\hline Identity \% & BLOSUM62 & EX & delta-V & delta-P & \# proteins & Length ave. \\
\hline$>99$ & 0.49 & 0.30 & 0.34 & 0.67 & 136 & 1532.7 \\
\hline 98-99 & 0.44 & 0.25 & 0.32 & 0.63 & 137 & 1464.0 \\
\hline 96-98 & 0.42 & 0.24 & 0.32 & 0.58 & 125 & 1539.4 \\
\hline $87-96$ & 0.38 & 0.20 & 0.31 & 0.55 & 57 & 1414.4 \\
\hline \multicolumn{7}{|c|}{ Protein length $>1102$ amino acid with gaps in alignment } \\
\hline$>99$ & 0.47 & 0.31 & 0.42 & 0.70 & 61 & 1659.3 \\
\hline 98-99 & 0.41 & 0.24 & 0.34 & 0.63 & 119 & 1855.8 \\
\hline 96-98 & 0.36 & 0.21 & 0.32 & 0.56 & 320 & 1792.7 \\
\hline $87-96$ & 0.33 & 0.19 & 0.31 & 0.50 & 437 & 1727.3 \\
\hline \multicolumn{7}{|c|}{ Protein length 200-1102 amino acid with no gaps in alignment } \\
\hline$>95$ & 0.43 & 0.25 & 0.32 & 0.63 & 6984 & 478.3 \\
\hline 90-95 & 0.39 & 0.20 & 0.31 & 0.55 & 1229 & 407.9 \\
\hline $80-90$ & 0.38 & 0.20 & 0.32 & 0.52 & 276 & 350.9 \\
\hline $60-80$ & 0.41 & 0.25 & 0.37 & 0.57 & 91 & 372.8 \\
\hline \multicolumn{7}{|c|}{ Protein length 200-1102 amino acid with gaps in alignment } \\
\hline$>95$ & 0.38 & 0.22 & 0.32 & 0.59 & 2001 & 601.1 \\
\hline $90-95$ & 0.33 & 0.19 & 0.31 & 0.51 & 1529 & 566.4 \\
\hline $80-90$ & 0.30 & 0.17 & 0.30 & 0.47 & 1050 & 489.1 \\
\hline $60-80$ & 0.33 & 0.20 & 0.33 & 0.48 & 467 & 447.1 \\
\hline
\end{tabular}

12 
Figure 1

Figure 1. Fraction of conservative substitutions in fixed changes in proteins of different evolutionary rates.

Shown are fractions of conservative changes in proteins of length $>1102$ aa $(A, B)$ or 200-1102 aa (C, D) with either no gaps in alignment $(A, C)$ and with gaps $(B, D)$. *: $P<0.05$. Chi-squared test.
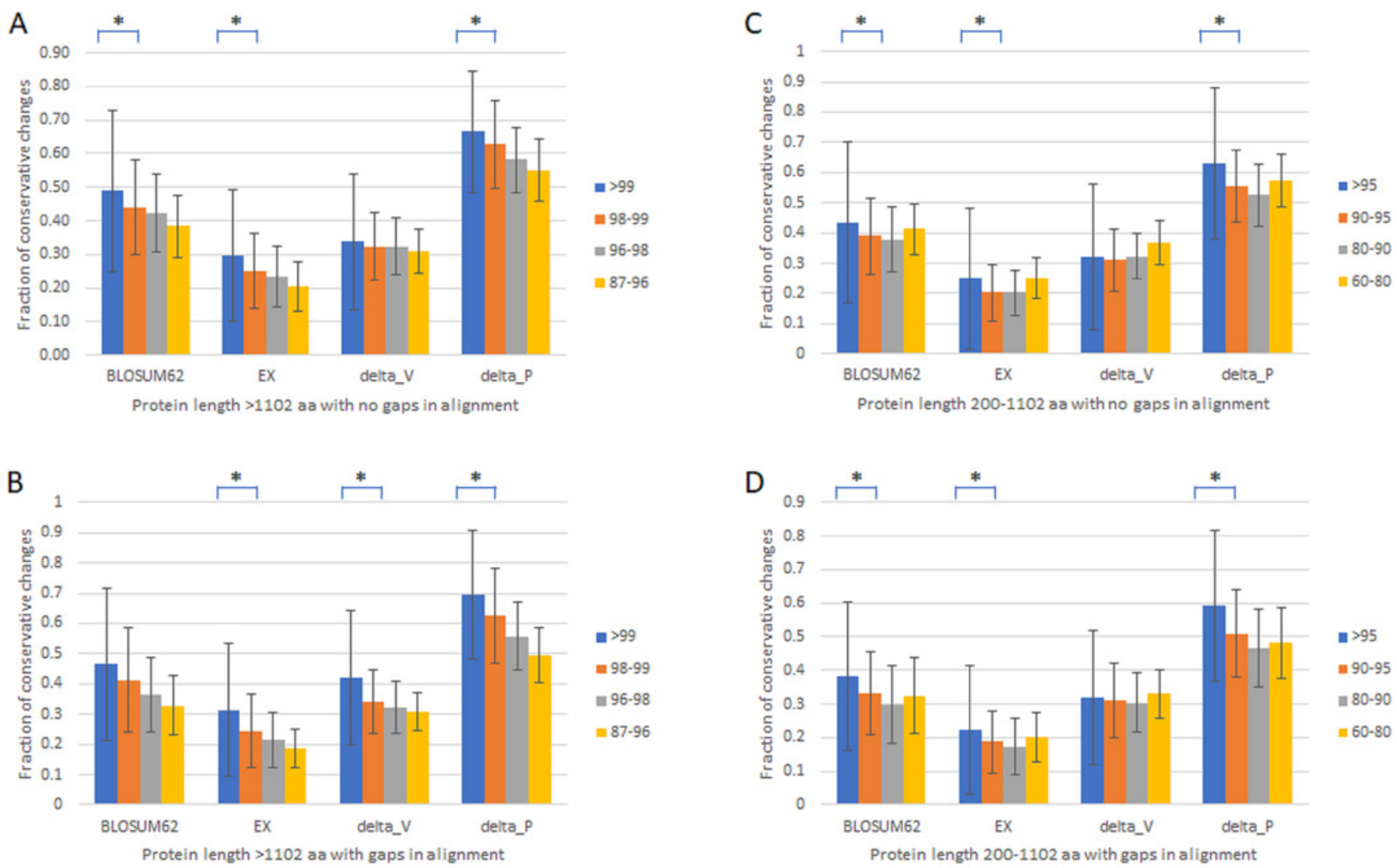
Figure 2

Figure 2. Fraction of conservative substitutions in standing missense substitutions in proteins of different evolutionary rates.

Missense SNPs from either the slow or the fast group of proteins were classified based on the scores in the four different matrixes as shown in A, B, C, and D. Shown are the fractions of each class. $* * *, \mathrm{P}<0.001$. $* *, \mathrm{P}<0.01$, Chi squared test.
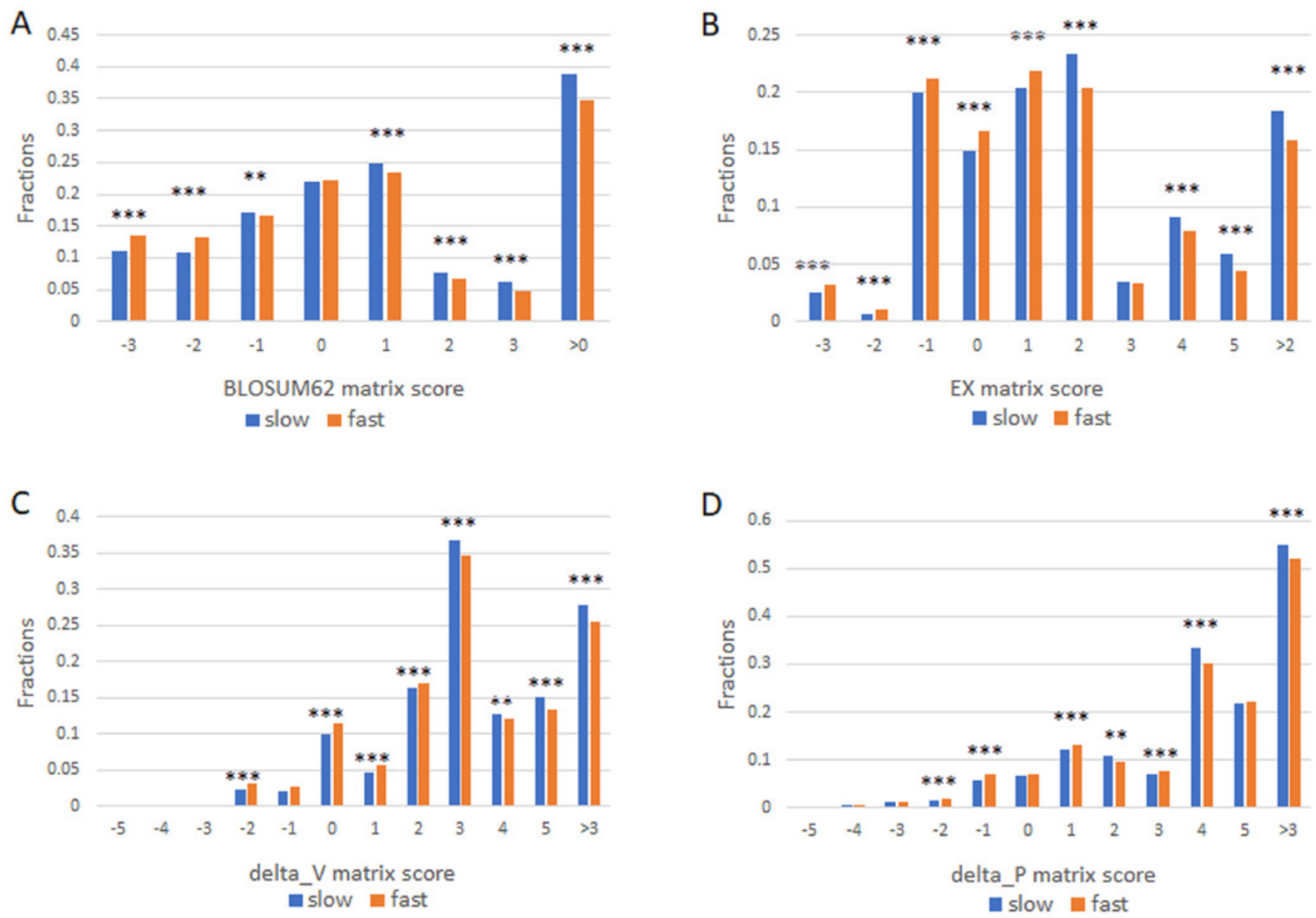


\section{Figure 3}

Figure 3. Fraction of conservative substitutions in missense SNPs with different MAFs in proteins of different evolutionary rates.

Figure 3. Fraction of conservative substitutions in missense SNPs with different MAFs in proteins of different evolutionary rates. SNPs from either fast or slowly evolving proteins were classified based on MAF values and the fractions of conservative changes in each class are shown. Statistical significance score in difference between slow and fast or between different MAF cutoffs are shown. ${ }^{* *}, \mathrm{P}<0.01$. Chi squared test. 


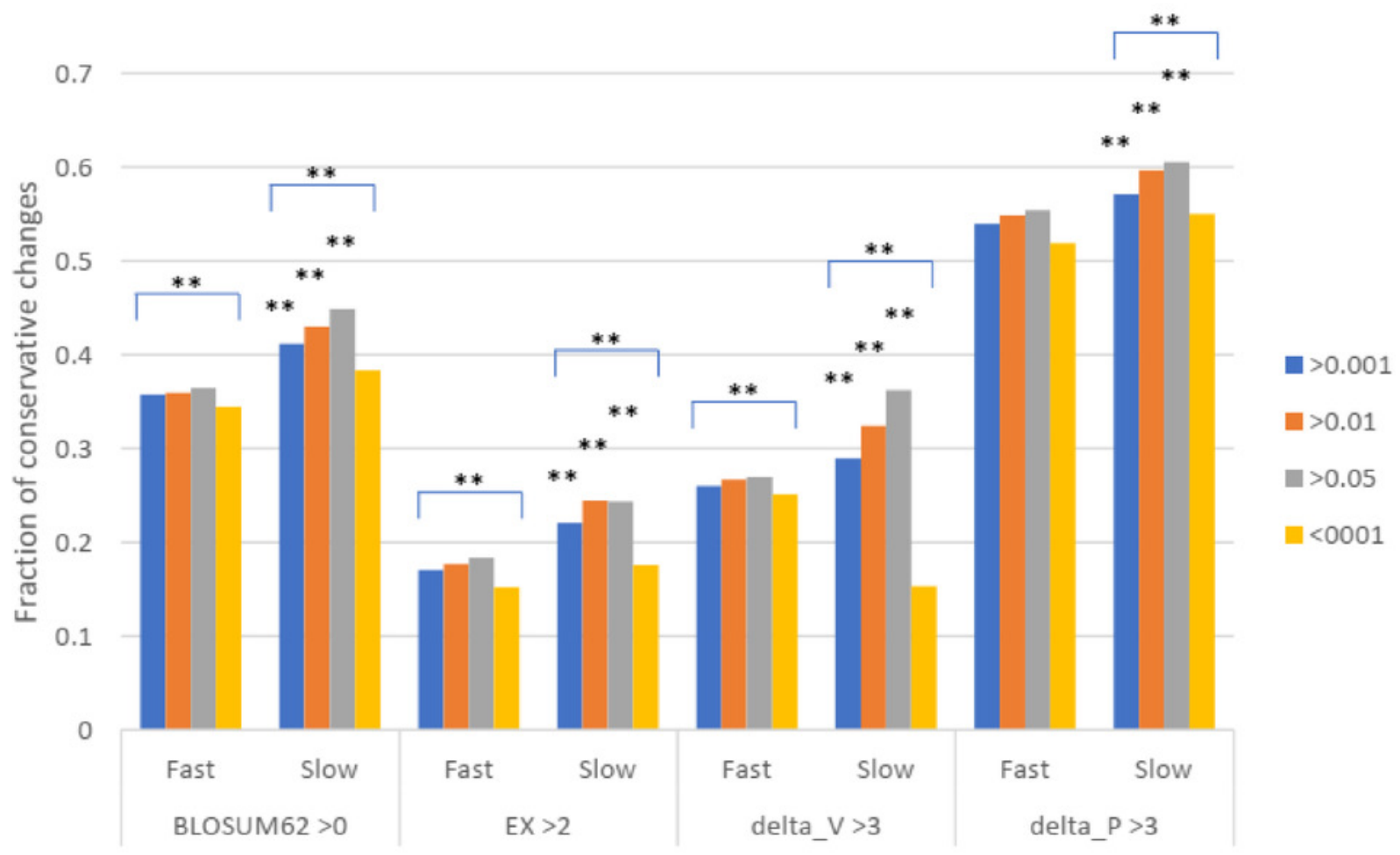




\section{Figure 4}

Figure 4. Illustration of non-conservative substitutions and mutation saturation in fast evolving proteins.

Three orthologous proteins from three different taxa are shown for either fast evolving (designated P1, P2, and P3) or slow evolving proteins (P4, P5, and P6). Nucleotide codon for residue $\mathrm{E}$ and its mutated codon in $\mathrm{P} 1, \mathrm{P} 3$, and $\mathrm{P} 6$ are also shown. A doublet mutation is found in the non-conservative substitution in P3 at time point 2 , whereas only a single mutation is found in the conservative substitution in P6. Saturation phase for fast evolving proteins includes time point 3 to 5 . The type of saturation we describe here is shown at time point 3 and 4 while the type seen in "long branch attraction" is shown at time point 5 . At the saturation phase, for the fast evolving protein $\mathrm{P} 1$ to have the new allele $\mathrm{D}$ at time point 4 or $\mathrm{S}$ at time point 5 is largely a matter of natural selection. 
Fast evolving proteins

$\begin{array}{llc} & \text { GAA } & \text { GAA } \\ \text { P1 } & \text { ACDEFGHI } & \text { ACDEFGHI } \\ \text { P2 } & \text { ACDEFGHI } & \text { ACDEFGHI } \\ \text { P3 } & \text { ACDEFGHI } & \text { ACDLFGHI } \\ & & \text { TTA }\end{array}$

GAC
ACDDFGHI
ACDEFGHI
ACDLFGHI
TTA

GAC
ACDDFGHI
ACDSFGHI
ACDLFGHI
TTA

TCC

ACDSFGHI

ACDSFGHI

ACDLFGHI

TTA

Slowly evolving proteins

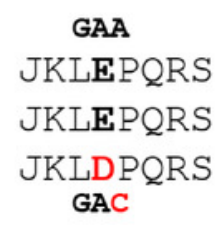
GAA
JKLEPQRS

P4 JKLEPQRS

P5 JKLEPQRS

P6 JKLEPQRS

GAA
JKLEPQRS
JKLEPQRS
JKLDPQRS
GAC

GAA

JKLEPQRS

JKLEPQRS

JKLDPQRS

GAC
GAA

JKLEPQRS

JKLEPQRS

JKLDPQRS

GAC

\begin{tabular}{llcccc}
1 & & & & \\
\hline 1 & 2 & 3 & 4 & 5
\end{tabular}

